\title{
Modelado matemático de caja blanca y negra en educación en ingeniería
}

\author{
Juan P. Cardona ${ }^{*}{ }^{*}$, John J. Leal ${ }^{2}$ y José E. Ustariz ${ }^{3}$ \\ (1) Universidad Cooperativa de Colombia, Facultad de Ingeniería, Av. Caracas No. 37-15, Bogotá D.C. Colombia. \\ (correo-e: juan.cardonag@campusucc.edu.co) \\ (2) Universidad Nacional de Colombia, Sede Palmira, Facultad de Ingeniería y Administración, Kr. 32 No 12-00, Palmira, \\ Valle del Cauca. (correo-e: jlealgom@unal.edu.co) \\ (3) Universidad Cooperativa de Colombia, Facultad de Ingeniería, Av. Caracas No. 37-15, Bogotá D.C. Colombia. \\ (correo-e: jose.ustariz@campusucc.edu.co)
}

* Autor a quien debe ser enviada la correspondencia.

Recibido Mar. 10, 2020; Aceptado May. 12, 2020; Versión final Jul. 20, 2020, Publicado Dic. 2020

\begin{abstract}
Resumen
En este estudio se presentan actividades desarrolladas en cursos de ingeniería con el objetivo de que los estudiantes utilicen procesos de modelado matemático para la comprensión de fenómenos físicos utilizando sistemas reales. La metodología del modelado en el aula se hizo siguiendo la propuesta de Kai Velten para ingenieros Se planteó el análisis del drenado de tanques con sección transversal variable en dos cursos distintos: 1) un curso en ecuaciones diferenciales ordinarias donde se establece una ecuación diferencial que modelara el drenaje, modelo de caja blanca (mecanicistas) basados en principios físicos y 2) un curso en medición de variables físicas donde se utiliza un ajuste polinómico de segundo orden por mínimos cuadrados, o modelo de caja negra (empírico). Se concluye que debido a la necesidad actual de solucionar problemas reales de ingeniería, se deben incluir técnicas de modelado matemático en los cursos de pregrado para que los estudiantes se habitúen a analizar problemáticas desde ésta perspectiva.
\end{abstract}

Palabras clave: modelado matemático; simulación; caja negra y blanca; educación; ingeniería

\section{Mathematical modeling of white and black box in engineering education}

\begin{abstract}
This research study presents activities on mathematical modeling processes that were developed in engineering courses to improve student understanding of physical phenomena using real systems. The modeling methodology performed in the classroom followed the proposal by Kai Velten for engineers. The analysis of tank drainage with cross-section variables were presented in two different courses: 1) a differential equations course where students established a differential equation modeling drainage by using a white box model (mechanistic) based on physical principles, and 2) a physical variables measuring course in which students used a second order polynomial adjustment by least squares or a black box model (empirical). Given the current need in engineering for solving real problems, it is concluded that mathematical modeling techniques must be included in undergraduate courses, such that students start getting accustomed to analyzing problems from this perspective.
\end{abstract}




\section{INTRODUCCIÓN}

La ingeniería es la ciencia para resolver problemas de la vida real, para ello los ingenieros desarrollan productos, procesos y servicios utilizando la tecnología disponible y su conocimiento científico. Además, ha evolucionado a través del tiempo desde sus inicios donde se realizaban tareas técnicas como soldar u operar máquinas, hasta la actualidad en la que uno de sus roles fundamentales es el diseño y construcción de elementos tecnológicos que den solución a problemáticas de la sociedad. (Venkateswarlu 2016 y Zawojewski 2008). Aún más de acuerdo con Cross (1994), los procesos de diseño en ingeniería utilizan cada vez más el modelado matemático como forma primaria; lo que permite al ingeniero desarrollar nuevas tecnologías, explorar campos emergentes especializados y predecir el comportamiento de un sistema antes de que realmente se construya. Igualmente, Cellier (1991) considera que el modelado matemático y la simulación se han convertido en tareas centrales en todas las disciplinas de la ingeniería y la ciencia dado que son las únicas técnicas disponibles que permiten analizar sistemas físicos con precisión y bajo condiciones experimentales variables; de la misma manera Mendible y Ortiz (2007) señala que en ingeniería el modelado matemático constituye una competencia profesional de innegable utilidad práctica.

Para Stadnicka et al. (2019) los ingenieros necesitan conocer un amplio espectro de técnicas y herramientas modernas para poder aplicarlas en su trabajo profesional. El modelado y la simulación son ejemplos de actividades que comúnmente realizan los ingenieros modernos, especialmente en el contexto del concepto de la Industria actual. Además, los lugares de trabajo de ingeniería modernos suelen utilizar prácticas de modelado matemático y simulación, junto con herramientas computacionales, para ayudar al análisis y diseño de sistemas (McKenna y Carberry, 2012). No obstante, a pesar de la importancia que presenta el modelado y la simulación en la práctica profesional de la ingeniería, es evidente la escasez de científicos e ingenieros que estén adecuadamente preparados para aprovechar o contribuir a estos desafíos científicos altamente interdisciplinarios y altamente computacionales (Shiflet y Shiflet, 2014; Yilmaz, 2012). Para Alpers (2017) dado que los ingenieros configuran y trabajan con modelos matemáticos que describen ciertas situaciones de interés, parece ser bastante obvio que las habilidades de modelado matemático deberían desempeñar un papel importante en la educación en ingeniería, sin embargo, no es claro en qué parte de los cursos de ingeniería se adquieren estas competencias, en temas de aplicación como estática o teoría de control, se introducen importantes cantidades de modelado (como fuerza, torque, tensión) y se presentan formas de configurar modelos y calcular cantidades interesantes para tales modelos, de modo que los estudiantes al menos "experimenten" el modelado matemático, pero solo trabajando constantemente en actividades de modelado los estudiantes pueden adquirir tales habilidades.

Para Magana (2017), los profesionales de la ciencia y la ingeniería han enfatizado en la necesidad de un enfoque nuevo y moderno para educar y capacitar a la próxima generación de profesionales de la ingeniería para complementar de manera efectiva los enfoques experimentales y teóricos de los procesos de descubrimiento e innovación. A si mismo Feisel y Rosa (2005) afirman que el objetivo general de la educación en ingeniería es preparar a los estudiantes para practicar ingeniería y, en particular, para lidiar con las fuerzas y los materiales de la naturaleza, más aún Li (2013) considera que el modelado matemático en la educación es la interfaz educativa más importante entre las matemáticas y la industria", de manera que es necesario realizar reformas en los planes de estudios de ingeniería de pregrado para producir ingenieros con habilidades que les permitan abordar los problemas del mundo real relevantes para la industria y la sociedad. El desarrollo de proyectos prácticos con asistencia tecnológica que implican experimentación, simulación de diseño y creación de prototipos transformarán a los graduados en profesionales con las habilidades necesarias para crear y avanzar en el conocimiento que cumpla con los estándares globales. (Venkateswarlu, 2017).

Magana (2017) presenta dos objetivos de las simulaciones en la educación en ingeniería: 1) usar simulaciones para identificar y describir los principios físicos fundamentales o comportamientos de dispositivos, materiales y otros artefactos; y 2) crear simulaciones para aplicar técnicas de modelado y computación para abordar las tareas de diseño de ingeniería. Por otra parte, Stadnicka et al. (2019) afirman que las simulaciones permiten presentar el mundo real con el uso de un modelo simplificado, que representa las acciones emprendidas en un proceso o los fenómenos que ocurren en el mismo. Los estudiantes, en muchos casos, no tienen posibilidad de observar cómo funciona un sistema real. Así que las simulaciones se pueden usar para analizar el comportamiento tales sistemas. Además, las empresas están interesadas en el desempeño de las simulaciones de procesos, para verificar las consecuencias de los cambios propuestos. También pueden contribuir significativamente con los resultados del aprendizaje, siempre que estén fuertemente relacionadas con los objetivos de los cursos de una manera clara y directa, de lo contrario serán una experiencia plana y poco interesante para estudiantes y profesores. Para Feisel y Rosa (2005) hoy en día, existen programas de software de simulación que emulan con precisión muchos procesos técnicos y físicos. Estos programas de software juegan un papel importante en la educación en ingeniería. De modo similar las tecnologías de simulación, permiten representar una realidad específica mediante un modelo lógico-matemático en el cual el 
estudiante puede interactuar con el modelo y explorar los efectos de sus decisiones en el funcionamiento del mismo. Ésta interacción consiste en cambiar parámetros de operación (lógicos o matemáticos) del modelo y analizar los resultados obtenidos luego de las modificaciones para entonces proponer alternativas de mejoramiento. Giraldo y Pinilla (2016).

La educación en ingeniería ha comenzado a reconocer la importancia de la formación de habilidades de modelado y simulación en las aulas, específicamente la Acreditation Board for Engineering and Technology,( ABET, 2017) en su informe "Criterios para la acreditación de programas de ingeniería", identifica que los estudiantes de ingeniería deben desarrollar entre otras capacidades las siguientes: aplicar los conocimientos de matemáticas, ciencias e ingeniería, diseñar y realizar experimentos, analizar e interpretar datos, diseñar un sistema, componente o proceso para cumplir las necesidades deseadas dentro de limitaciones realistas, tales como economía, medio ambiente, social, política, ética, salud y seguridad, capacidad de fabricación y sostenibilidad, identificar, formular y resolver problemas de ingeniería, y utilizar las técnicas, habilidades y herramientas de ingeniería moderna necesarias para la práctica de la ingeniería.

Igualmente, la Sociedad Estadounidense para la Educación en Ingeniería (ASEE, 2013) en el documento "Transformando la Educación de Pregrado en Ingeniería" se identifican una serie de habilidades que se deben desarrollar en las aulas de ingeniería y que los futuros profesionales las deben aplicar para satisfacer las necesidades de la industria en el siglo XXI, dentro de ellas están: el uso de nuevas tecnologías y herramientas de ingeniería modernas necesarias para la práctica de ingeniería, conocimiento de ciencias básicas de ingeniería y capacidad de implementación para aplicarlas en el mundo real, interpretación y visualización de datos, comprensión del diseño en ingeniería, sólidos fundamentos de ciencias físicas y ciencias de la ingeniería, capacidad para identificar, formular y resolver problemas de ingeniería. Además, promueve los cambios en los planes de estudio, la pedagogía y la cultura académica necesarios para desarrollar las habilidades en la próxima generación de ingenieros. De la misma manera, la Asociación Iberoamericana de Instituciones de Enseñanza de la Ingeniería (ASIBEI, 2016) en su informe "Competencias y perfil del ingeniero iberoamericano, formación de profesores y desarrollo tecnológico e innovación" identifica dentro de las características del ingeniero lberoamericano: La habilidad de analizar, modelar, experimentar y resolver problemas de diseño, de soluciones abiertas y de enfoque multidisciplinario y la capacidad de utilizar eficientemente el creciente desarrollo de las telecomunicaciones y las herramientas informáticas.

Por otra parte en Colombia el Ministerio de Educación Nacional (MEN, 2003) como responsable de la formulación de la política educativa en la formación de ingenieros plantea el desarrollo de aspectos curriculares que desarrollen la conceptualización, diseño, experimentación, formulación de modelos y desarrollos tecnológicos propios de cada especialidad en ingeniería, también algunas instituciones de educación superior como la Universidad Cooperativa de Colombia plantea en los programas de ingeniería como competencia: "modelar matemáticamente procesos a partir de la representación de los fenómenos naturales para resolver problemas relacionados con materiales, estructuras, máquinas, dispositivos y sistemas que en forma segura logren el objetivo esperado".

En Frejd y Bergsten (2015) se muestra una investigación detallada en la que se presentan varias experiencias de modeladores profesionales en distintas áreas quienes emplean las técnicas de modelado matemático de caja blanca, caja gris o modelos híbridos y modelos de caja negra, allí se resaltan las ventajas y desventajas de cada uno de los métodos empleados. Éste tipo de modelos también se han utilizado en los trabajos de Zendehboudi, Nima y Ali (2018), Nugroho y Akmeliawati (2018), Wang et al. (2015), Casoli (2011) y Van Can (1998) entre otros. En general los autores modelaron, simularon y compararon los resultados de los diferentes tipos de modelos con datos reales para determinar cuál era el "mejor" modelo matemático que describía su sistema físico.

Frejd y Bergsten (2015) proponen una discusión para incorporar las técnicas de modelado matemática en la escuela utilizando la trasposición didáctica (Chevallard, 1991), por otra parte, Leal et al. (2015) propone que la educación en ingeniería debe estar fundamentada en el quehacer profesional de los ingenieros que consiste en el estudio de los sistemas físicos reales utilizando modelado matemático. Teniendo en cuenta los argumentos expuestos anteriormente, nos hemos propuesto promover en los estudiantes de ingeniería el planteamiento y la solución a problemas reales utilizando las técnicas de modelado matemático de caja blanca y caja negra.

En la Figura 1 se muestra el análisis bibliométrico de la base de datos SCOPUS, en el cual se encuentran 160 documentos que muestran la relación entre educación en ingeniería y modelos de caja negra y blanca, en donde se puede apreciar que la relación directa es escasa, lo que indica una desarticulación entre el proceso de formación de los ingenieros en modelado matemático y su desarrollo profesional. 


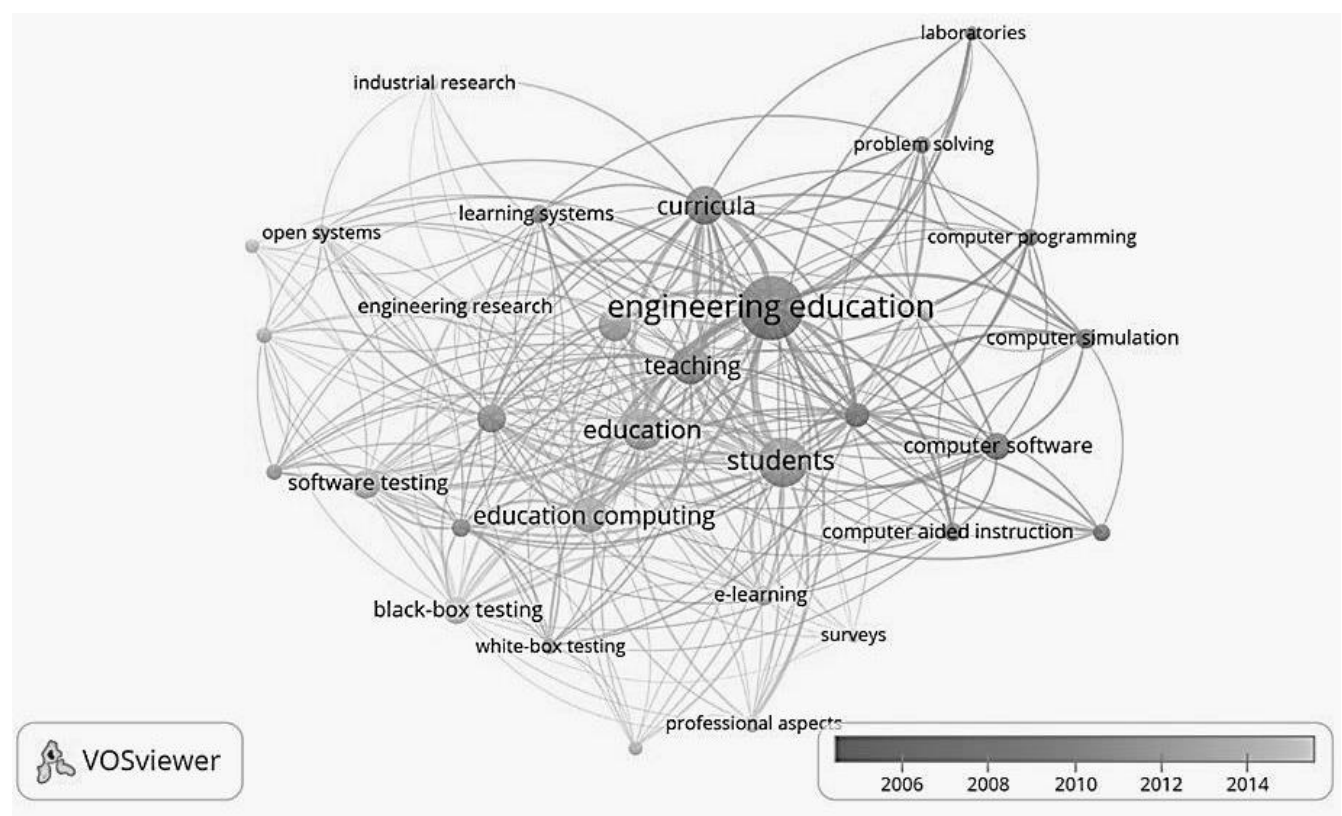

Fig. 1: Red bibliométrica educación en ingeniería y modelos matemáticos de caja blanca y negra.

\section{DEFINICIONES Y TERMINOLOGÍA EN MODELADO MATEMÁTICO}

Con el objeto de contextualizar el trabajo sobre el modelado matemático en la formación de ingenieros, se presentan a continuación los términos básicos que sirven de base a la discusión teórica:

\section{Sistema}

Para Zeigler (2019), un sistema es una parte de la realidad entendida como una fuente potencial de datos. Por otra parte, Fritzson (2011), considera un sistema como un objeto o una colección de objetos cuyas propiedades queremos estudiar. La finalidad es analizarlo o construirlo. Además, un sistema se caracteriza por el hecho de que el modelador puede definir sus límites, sus atributos y sus interacciones con el entorno en la medida en que el modelo resultante puede satisfacer los objetivos del modelador. Nirmala (2002). Ciertamente, las variables de entrada y salida, parámetros y constantes son atributos que se deben reconocer al modelar matemáticamente un sistema. (Fritzson 2011; Nirmala 2002; Edwards y Hamson,1989)

\section{Experimento}

Cellier (1991) argumenta que un experimento es el proceso de extracción de datos de un sistema mediante la manipulación de sus variables de entrada. Para poder realizar un experimento sobre un sistema, éste debe de ser tanto controlable como observable. De tal forma que se aplica un conjunto de condiciones externas a las variables de entrada y se observan y miden sus variables de salida.

\section{Modelo}

Para Minski (1965) $A^{*}$ es un modelo de un objeto $A$ en la medida que un observador $B$ puede usar $A^{*}$ para responder preguntas que le interesan sobre A. Según Shier (1999) un modelo se puede definir como una representación simplificada de ciertos aspectos de un sistema real, se crea con el objetivo de obtener nuevos conocimientos sobre el mundo real mediante la investigación de las propiedades y las implicaciones del modelo. De acuerdo con Cellier (1991) un modelo (M) de un sistema (S) y un experimento (E) es cualquier cosa a la que se pueda aplicar E para responder preguntas sobre S".

\section{Modelo Matemático}

Fritzon (2011), plantea que un modelo matemático es una descripción de un sistema donde las relaciones entre las variables del sistema se expresan de forma matemática. Las variables pueden ser cantidades medibles, tales como el tamaño, la longitud, el peso, la temperatura, el nivel de desempleo, el flujo de información, la velocidad medida en bits por segundo, etc. La mayoría de las leyes de la naturaleza son modelos matemáticos en este sentido. Por ejemplo, la Ley de Ohm describe la relación entre la corriente y la caída de tensión en una resistencia; las Leyes de Newton describen relaciones entre la velocidad, la aceleración, la masa, la fuerza, etc. Igualmente, Edwards y Hamson (1996) mencionan que en la formulación 
de un modelo matemático se deben identificar los siguientes aspectos: 1) Constantes: cantidades cuyos valores no podemos cambiar. Estas pueden ser constantes matemáticas, o constantes físicas, como la aceleración debida a la gravedad o la velocidad de la luz. 2) Parámetros: cantidades que son constantes para una aplicación particular de un modelo, pero pueden tener valores diferentes para otra aplicación del mismo modelo. Por ejemplo, las dimensiones de una habitación, la densidad de un fluido, el tiempo medio entre llegadas de un servicio de autobús.3) Variables de entrada: cantidades que determinan evaluaciones dentro del modelo. 4) Variables de salida: cantidades que son consecuencia de valores dados de variables y parámetros de entrada y que no se pueden dar valores arbitrarios. Estos representan el resultado de un modelo.

Además, Velten (2009) definió un modelo matemático como una tripleta $(\mathrm{S}, \mathrm{Q}, \mathrm{M})$ donde $\mathrm{S}$ es un sistema, $\mathrm{Q}$ es una pregunta relacionada con $S$ y $M$ es un conjunto de sentencias matemáticas $M=\{1,2, \ldots, n\}$ que se puede usar para contestar Q. De acuerdo con Preziosi y Bellomo (1994) un modelo matemático está conformado un conjunto de ecuaciones que se pueden usar para calcular la evolución espacio-temporal de un sistema físico. Para Cellier (1991) un modelo matemático es una subclase de modelos que se pueden codificar como programas de computador.

\section{Simulación}

De acuerdo con Cellier (1991) una simulación es un experimento realizado sobre un modelo, por otra parte, Fritzson (2011) afirma que, si el modelo matemático se representa en forma ejecutable en un computador, las simulaciones se pueden realizar mediante experimentos numéricos, o en casos no numéricos mediante experimentos computados. Esta es una forma simple y segura de efectuar experimentos, con la ventaja añadida de que esencialmente todas las variables del modelo son observables y controlables. Sin embargo, el valor de los resultados de simulación es completamente dependiente de que tan bien está formulado el modelo representa al sistema real con respecto a las cuestiones a las que la simulación tiene que responder. Excepto por la experimentación, la simulación es la única técnica que es aplicable de forma general para el análisis del comportamiento de sistemas.

\section{Modelado}

Según (Zeigler 2019; Nirmala 2002) modelado significa el proceso de organización y aplicación del conocimiento para describir el desempeño de un sistema real para lograr ciertos objetivos. Para Shiflet (2002) el modelado es la aplicación de métodos para analizar complejos problemas del mundo real para hacer predicciones sobre qué podría suceder con varias acciones. De acuerdo con (Cellier 1991; Nirmala 2002) los modelos matemáticos van desde los modelos de "caja blanca" bien definidos y rigurosos hasta los modelos de "caja negra" mal definidos y empíricos. Un modelo matemático de caja blanca o mecanicista se basa en el enfoque deductivo o teórico. Aquí, las teorías y principios fundamentales que gobiernan el sistema junto con los supuestos simplificadores se utilizan para derivar relaciones matemáticas entre las variables que se saben o que se suponen son significativas. El modelo resultante se puede calibrar con datos históricos del sistema real y se puede validar con datos adicionales. Las predicciones se pueden hacer con confianza predefinida y se pueden utilizar para el diseño de sistemas, además reflejan cómo los cambios en el rendimiento del sistema se relacionan con los cambios en las entradas.

Por el contrario, un modelo matemático de caja negra o empírico se basa en un enfoque inductivo es decir en datos los cuales se utilizan para desarrollar relaciones entre variables que se consideran significativas en el sistema en estudio. Las herramientas estadísticas se utilizan a menudo en este proceso para garantizar la validez de las predicciones para el sistema real. El modelo resultante se considera una "caja negra", que refleja sólo los cambios que podrían esperarse en el rendimiento del sistema debido a cambios en las entradas. A pesar de que el valor de utilidad de este enfoque se limita a las predicciones, ha demostrado ser útil en el caso de sistemas complejos donde la ciencia subyacente no se comprende bien. Otros autores como Nugroho y Akmeliawati (2018) definen el tercer tipo de modelo denominado modelo de caja gris que requiere el uso de datos empíricos del sistema fusionados con la estructura del modelo basado en leyes físicas o geométricas para identificar los parámetros del modelo necesarios para estimar comportamientos específicos propios del funcionamiento del sistema.

Por otra parte, Velten (2009), propone una clasificación de los modelos matemáticos entre modelos de caja blanca y negra tal como se muestra en la Figura 2. En un extremo del espectro se ubican los sistemas psicológicos y sociales que constituyen los modelos de "caja negra" estos modelos se pueden usar para hacer predicciones más o menos confiables basadas en datos. Los sistemas mecánicos, los circuitos eléctricos, se ubican en el otro lado del espectro conformando los sistemas de caja blanca, ellos se entienden muy bien en términos de modelos mecanicistas, En el extremo de la caja blanca del espectro, se pueden aplicar modelos matemáticos para diseñar, probar y optimizar sistemas y procesos en la computadora antes de que se realicen 
físicamente. Los modelos de caja blanca están fundamentados en leyes físicas. Se debe tener en cuenta que las tres dimensiones de un modelo matemático (S, Q, M) se pueden ver en la Figura 2: los sistemas (S) se clasifican en la parte superior de la barra, justo debajo de la barra hay una lista de objetivos que los modelos matemáticos en cada uno de los segmentos pueden tener (que es $Q$ ), y en el extremo inferior hay las correspondientes estructuras matemáticas $(M)$ que van desde las estructuras de ecuaciones algebraicas (AE) hasta las estructuras de las ecuaciones diferenciales ordinarias o parciales (DE).

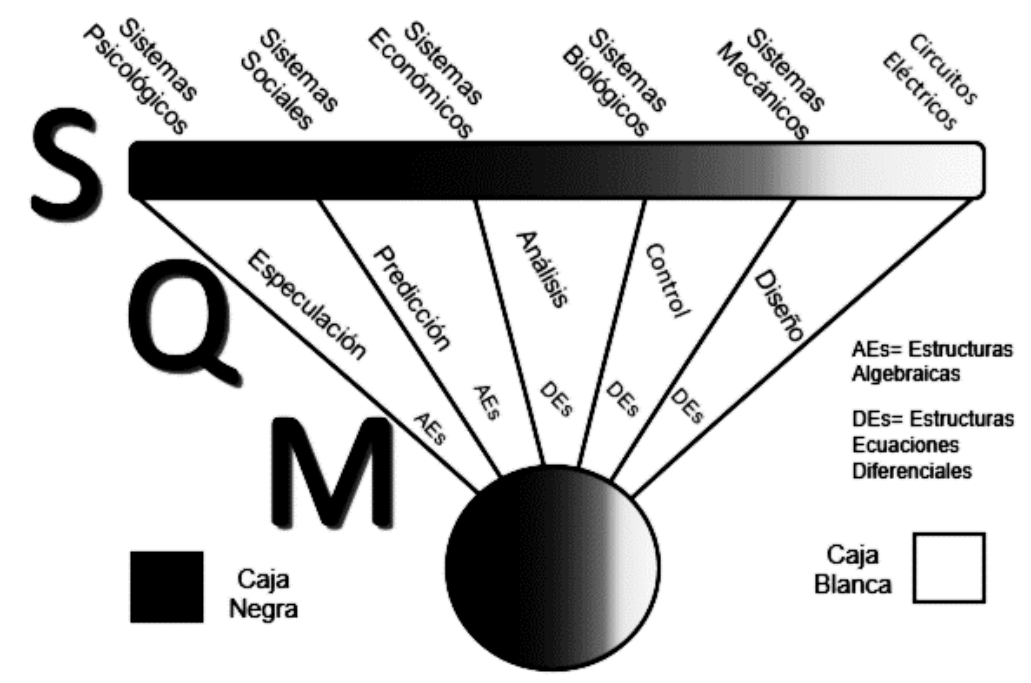

Fig.2: Espacio de trabajo S, Q, M de caja blanca y negra, Adaptada de Velten (2009)

\section{MODELOS MATEMÁTICOS DE CAJA BLANCA}

A continuación, se describen cada una de las etapas de modelado matemático de caja blanca consideradas por Velten (2009), y se presentan una serie de tareas y aclaraciones sobre cada una de ellas propuestas por los autores para la implementación en el aula.

\section{Definiciones}

En esta etapa el futuro ingeniero debe caracterizar el sistema a estudiar, esto implica: identificarlo, definirlo apropiadamente, reconocer sus límites, describir su funcionamiento, determinar el/los materiales con el cual está construido, tener claridad sobre su dimensionamiento geométrico. Además, debe generar la pregunta que va a responder del sistema por medio del modelado matemático. A menudo, se crea un modelo esquemático, gráfico o pictográfico del sistema (2D o 3D) para visualizarlo apropiadamente. Al finalizar esta etapa el estudiante de ingeniería debe tener claridad sobre el funcionamiento del sistema.

\section{Análisis del sistema}

El estudiante de ingeniería debe identificar las partes del sistema que son relevantes para responder la pregunta $(Q)$ del modelado matemático, para ello debe: definir las variables de entrada y salida, constantes y parámetros del sistema, definir de forma apropiada el sistema de unidades (Internacional o inglés) que va a utilizar en el desarrollo del modelo matemático, reconocer el tipo de relación matemática (directa o inversa) entre las variables principales definidas del modelo. Otra parte muy útil e importante es preparar una lista de todas las variables junto con sus dimensiones fundamentales (masa $M$, longitud $L$ y tiempo $T$ ), esto puede ayudar a verificar la consistencia entre las variables y las ecuaciones, la resolución de problemas y determinar los resultados. Cada problema implica una serie de diferentes "factores" que pueden influir en la solución. En la primera etapa de la construcción de modelos, necesitamos una lista de estos factores.

\section{Modelado}

El estudiante de ingeniería identifica teorías, leyes y principios fundamentales que rigen el comportamiento del sistema y que son aplicables al mismo y además pueden ayudar a conseguir el objetivo de modelado matemático. Por ejemplo, leyes de Newton, conservación de energía, leyes de Kirchoff, entre otras. A partir de allí se deben derivar relaciones entre las variables de importancia y relevancia del sistema, de esta forma se transforma esencialmente el sistema real en una representación matemática que se debe analizar a la luz de las técnicas matemáticas existentes. Dicho proceso se conoce como matematización, Según Alma y García (2011), matematizar el mundo real, implica que el estudiante debe: interpretar datos, establecer 
relaciones y conexiones; poner en juego conceptos; analizar regularidades; establecer patrones de cambio; encontrar, elaborar, diseñar y/o construir modelos. En esta etapa además se debe revisar que el modelo matemático es dimensionalmente correcto, verificando la igualdad dimensional en el mismo.

\section{Simulación}

Aquí el estudiante de ingeniería aplica el modelo matemático al sistema en estudio, de tal forma que es necesario la utilización de técnicas y procedimientos matemáticos estándar para "resolver" el modelo y obtener los resultados deseados, en esta etapa se puede utilizar simulación numérica por computador mediante software especializado para generar datos sobre el sistema en estudio. La conveniencia de la representación matemática es que el modelo resultante puede analizarse solo, sin tener en cuenta el sistema real, temporalmente. El análisis se realiza de acuerdo con las reglas de las matemáticas, y el sistema no tiene nada que ver con ese proceso. El tipo de análisis que se utilizará será dictado por las relaciones derivadas en el paso anterior. Las técnicas analíticas generalizadas pueden caer en categorías algebraicas, diferenciales o numéricas.

\section{Validación}

En esta etapa el estudiante de ingeniería debe comparar los resultados del modelo matemático obtenidos en la etapa de simulación y compararlos matemática o estadísticamente con los resultados obtenidos sobre el sistema real, para ello es importante identificar las pruebas que se pueden realizar sobre el sistema para validar el modelo matemático, Para obtener datos del sistema real el estudiante de ingeniería debe realizar prácticas experimentales sobre el sistema y/o apoyarse en software especializado. El rendimiento del modelo se compara con el sistema real para garantizar que los resultados del modelado se cumplan satisfactoriamente. Incluso si los teoremas y principios fundamentales utilizados para construir el modelo describen el sistema de manera veraz, su rendimiento podría desviarse del sistema real debido a las suposiciones y simplificaciones inherentes hechas. Una vez validado el modelo matemático se puede utilizar para realizar predicciones y sacar conclusiones. En el proceso de calibración, los datos observados previamente del sistema real se utilizan como un conjunto de "entrenamiento". El modelo se ejecuta repetidamente, ajustando los parámetros del modelo por ensayo y error (dentro de rangos razonables) hasta que sus predicciones en condiciones similares coincidan con el conjunto de datos de entrenamiento según los objetivos y criterios de rendimiento establecidos. validación del modelo. A menos que un modelo esté bien calibrado y validado, su aceptabilidad seguirá siendo limitada y cuestionable. No hay puntos de referencia estándar para demostrar la validez de los modelos, porque los modelos deben estar vinculados a los sistemas que están diseñados para representar.

\section{MODELOS MATEMÁTICOS DE CAJA NEGRA}

Se pueden utilizar en una gran diversidad de fenómenos naturales y sociales, sin necesidad de conocer las relaciones entre los diferentes componentes de un sistema, de tal forma que si alguna parte de éste tiene cambios significativos no es posible determinar con precisión cuales fueron esas variaciones, el análisis es una relación de causa- efecto de variables matemáticas de entrada y salida. Los datos se analizan utilizando técnicas estadísticas y ajustes de curvas.

\section{Modelado matemático}

Es una metodología mediante la cual se generan modelos matemáticos para tratar de predecir el comportamiento de un sistema real. Dependiendo del tipo de modelo matemático que se formule, se requieren tipos de análisis particular. La metodología de modelado utilizando modelos matemáticos de caja blanca y negra tienen los siguientes aspectos coincidentes: Permiten obtener soluciones aproximadas para hacer predicciones sobre el funcionamiento de los sistemas, se utilizan técnicas estadísticas para la validación de los resultados y es necesario utilizar software. En los modelos de caja negra no es necesario conocer cómo funciona exactamente un sistema, a diferencia de los modelos de caja blanca, en los cuales es fundamental la comprensión de las leyes o principios físicos que rigen el comportamiento y la estructuración del mismo. Otra diferencia esencial, es el conocimiento matemático que se requiere para resolver cada tipo de modelo, puesto que en los modelos de caja blanca resultan ecuaciones mucho más complejas que en los modelos de caja negra.

\section{Implementación de la metodología de modelado matemático}

Para cumplir el objetivo propuesto en el que los estudiantes utilicen procesos de modelado matemático para la comprensión de fenómenos físicos utilizando sistemas reales, se propuso un problema transversal que permitiera utilizar los dos tipos de modelos en cada curso y de acuerdo con su nivel de formación (caja negra 
y blanca), y se trataba de determinar el tiempo de vaciado de un tanque de sección transversal variable. El problema se propuso en dos cursos distintos cada uno con treinta estudiantes de ingeniería de la Universidad Cooperativa de Colombia. Uno de ellos es el curso de medición de variables físicas, introductorio en el segundo semestre en el cual los estudiantes están apenas incursionando tanto en el manejo de herramientas matemáticas como en el uso de herramientas tecnológicas, quienes utilizaron el modelo de caja negra. El otro es un curso de ecuaciones diferenciales ordinarias, en el cual los estudiantes tienen fundamentos de matemáticas y física, han manejado distintas herramientas de software y de antemano han trabajado el software Matlab y su entorno gráfico Simulink, a éste grupo se le propuso utilizar un modelo de caja blanca.

Al curso de medición de variables físicas, se le planteó que hicieran el experimento y utilizando el software TRACKER adquirieran datos para luego ajustarlos a un polinomio por mínimos cuadrados, y luego determinaran con éste modelo el tiempo de vaciado del tanque, posteriormente hicieron la comparación porcentual con el valor teórico para validar el modelo. Por otra parte, los estudiantes del curso de ecuaciones diferenciales formularon un modelo matemático de caja blanca del sistema, definieron las variables de entrada y salida, establecieron relaciones entre tales variables utilizando ecuaciones diferenciales y basados en leyes físicas, luego solucionaron el modelo utilizando las técnicas empleadas para tal fin y finalmente validaron el modelo con los datos experimentales obtenidos mediante el software TRACKER.

\section{RESULTADOS}

A continuación, se presentan los resultados obtenidos por los estudiantes en el desarrollo de los dos tipos de modelos matemáticos.

\section{Análisis de un modelo matemático de caja blanca.}

En esta sección se presentan los resultados obtenidos por los estudiantes en cada una de las fases de modelado matemático en ingeniería.

\section{Definición del Sistema.}

El sistema que va ser modelado matemáticamente lo conforma un tanque de sección transversal rectangular variable, el cual inicialmente contiene agua a una altura de $12 \mathrm{~cm}$. El agua es drenada por un agujero circular de $1 \mathrm{~cm}$ de diámetro que se encuentra ubicado en la base del tanque, las dimensiones del mismo se muestran en la Figura 3a. El material del tanque es acrílico transparente el cual permite la toma de datos experimentales, el sistema físico se muestra en la Figura 3b. El objetivo del modelado matemático es generar un modelo por medio de ecuaciones diferenciales ordinarias que permita determinar el nivel del agua para un tiempo determinado y que responda a la siguiente pregunta: ¿Cuál es el tiempo de drenado del tanque?

\section{Análisis del sistema.}

En el modelo matemático del drenado del tanque los estudiantes reconocen los factores (Variables, constantes, parámetros) que intervienen en el mismo, al igual que el símbolo algebraico de cada uno de ellos y las unidades de medida utilizadas en el modelo matemático, dicha información se consigna en un formato como el que se muestra en la Tabla 1.

Tabla 1: Variables, parámetros y constantes del modelo matemático.

\begin{tabular}{|l|l|l|l|l|}
\hline Descripción & Tipo & Símbolo & Dimensiones & Unidades \\
\hline Área de drenado & Parámetro & $\mathrm{a}$ & $\mathrm{L}^{2}$ & $\mathrm{~cm}^{2}$ \\
\hline Área variable del tanque & Variable & $\mathrm{A}(\mathrm{y})$ & $\mathrm{L}^{2}$ & $\mathrm{~cm}^{2}$ \\
\hline Gravedad & Constante & $\mathrm{g}=980$ & $\mathrm{LT}^{-2}$ & $\mathrm{~cm}^{2}$ \\
\hline Tiempo de drenado del tanque & Variable de salida & $\mathrm{t}$ & $\mathrm{T}$ & $\mathrm{s}$ \\
\hline Altura del agua en el tanque & Variable de Entrada & $\mathrm{y}$ & $\mathrm{L}$ & $\mathrm{cm}$ \\
\hline
\end{tabular}

Adicionalmente es necesario realizar suposiciones de cómo es la relación entre las variables de entrada y salida del sistema, conociendo el funcionamiento del mismo, para este caso la relación entre el tiempo de drenado y la altura del agua en el tanque es Inversa.

\section{Principios físicos de modelado}

A continuación, se muestran los resultados del grupo de estudiantes de ecuaciones diferenciales con respecto a los principios físicos de funcionamiento del sistema y matematización de los mismos. 


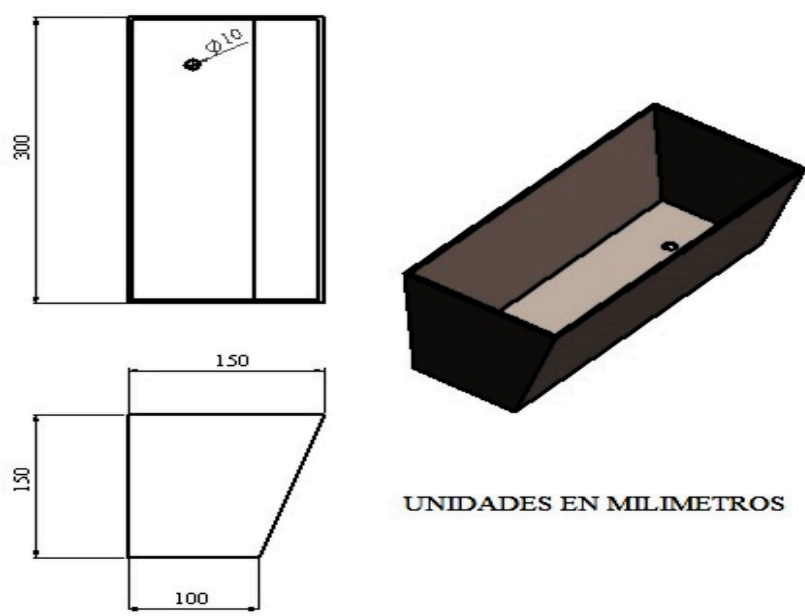

(a)

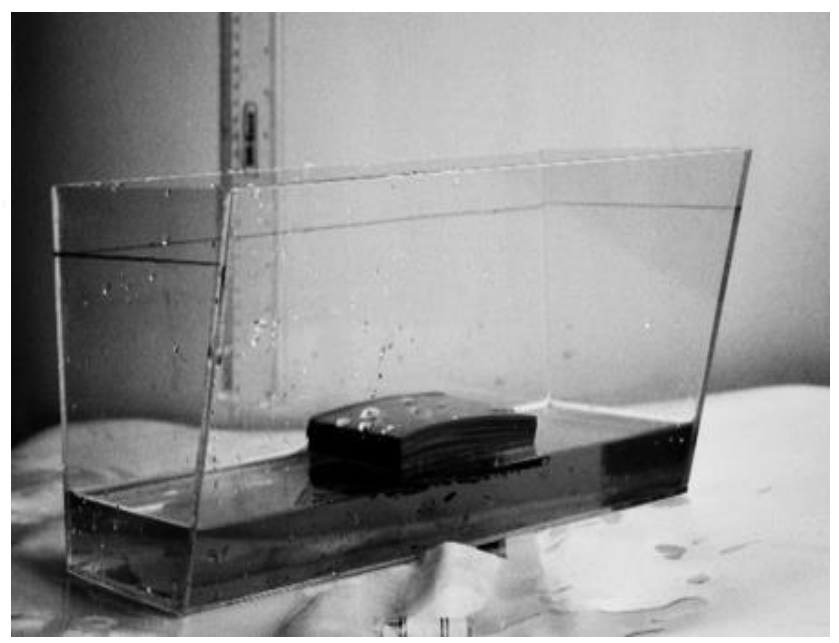

(b)

Fig. 3: a. Dimensiones geométricas del sistema. b. Sistema físico

\section{Conservación de la Energía (Principio de Bernoulli)}

El principio de Bernoulli es una consecuencia de la conservación de la energía en los líquidos en movimiento. Establece que, en un líquido incompresible y no viscoso, la suma de la presión hidrostática $(P)$, la energía cinética por unidad de volumen $\left(\rho v^{2}\right)$, y la energía potencial gravitatoria por unidad de volumen ( $\rho g h$ ) es constante a lo largo de todo el recorrido del fluido, como se puede apreciar en la ecuación 1.

$$
\mathrm{P}+\frac{1}{2} \rho v_{1}^{2}+\rho g h_{1}=\mathrm{P}+\frac{1}{2} \rho v_{2}^{2}+\rho g h_{2}
$$

Dónde: $\mathrm{v}=$ velocidad del fluido en la sección considerada. $\mathrm{g}=$ aceleración gravedad $\mathrm{h}=$ altura en la dirección de la gravedad desde una cota de referencia. $P=$ presión a lo largo de la línea de corriente. $\rho=$ densidad del fluido. Los subíndices 1 y 2 son puntos diferentes en el fluido.

\section{Principio de Torricelli}

El principio de Torricelli afirma que la velocidad del líquido que sale por el orificio en la pared de un tanque o recipiente, es idéntica a la que adquiere un objeto que se deja caer libremente desde una altura igual a la de la superficie libre del líquido hasta el orificio, supone que las pérdidas por viscosidad son despreciables, al igual que en la caída libre se supone que la fricción debida al aire que circunda al objeto que cae es insignificante. La suposición anterior es razonable en la mayoría de los casos y además implica la conservación de la energía mecánica del sistema $E_{m}$, de donde $E_{m 1}=E_{m 2}$, en donde $E_{c}$ es la energía cinética del sistema y $E_{p}$ es la energía potencial del sistema, los subíndices 1 y 2 son puntos diferentes en el fluido, ver la ecuación 2:

$$
\mathrm{E}_{\mathrm{c} 1}+\mathrm{E}_{\mathrm{p} 1}=\mathrm{E}_{\mathrm{c} 2}+\mathrm{E}_{\mathrm{p} 2}
$$

Reemplazando los correspondientes valores de energías cinética y potencial, se obtiene la ecuación 3:

$$
0+m g y=\frac{1}{2} m v^{2}+0
$$

Y finalmente, al despejar la velocidad v se obtiene la ecuación 4:

$$
v=\sqrt{2 g y}=\sqrt{2 g}(y)^{1 / 2}
$$

\section{Permanencia de Caudal}

Para determinar el caudal de fluido que permanece en el tanque $Q_{p}$ se realiza la resta del fluido que entra $Q_{E}$ y el que sale del mismo $Q_{s}$, así $Q_{p}=Q_{E}-Q_{s}$ En donde: $Q_{p}=d V / d t$, donde $V$ es el volumen de agua del tanque, y $Q_{E}=0$ y $Q_{s}=v a$, donde $v$ se obtuvo en la ecuación 4 y al reemplazarlo se obtiene la ecuación 5: 


$$
\frac{d V}{d t}=-a \sqrt{2 g}(y)^{1 / 2}
$$

Finalmente, después de despejar el diferencial de volumen $\mathrm{dV}=\mathrm{A}(\mathrm{y}) \mathrm{dy}$, y reemplazarlo en la ecuación 5 , se obtiene la ecuación 6 , en donde $\mathrm{A}(\mathrm{y})$ representa el área de la sección transversal a una altura y:

$$
\frac{d y}{d t}=\frac{-a}{A(y)} \sqrt{2 g}(y)^{1 / 2}
$$

\section{Modelo matemático}

La ecuación 6 corresponde con el modelo matemático general para el drenado de tanques, en éste caso se considera que el área de la sección transversal del drenado es $\pi / 4 \mathrm{~cm}^{2}$ y el valor de la constante $\sqrt{2} g=$ 44,27. Para el cálculo del área variable del tanque $A(y)$ en la Figura 3a se observa que las secciones transversales del tanque son rectángulos de ancho variable $x$, que se hallan utilizando la ecuación de la línea recta que une los puntos $\mathrm{A}$ y $B$ de la Figura 4 , de esta forma se obtiene la ecuación 7 . Con el área variable de los rectángulos $A(y)$ obtenidos a partir de la ecuación 7 se obtiene la ecuación 8 .

$$
\begin{aligned}
& x=\frac{y+30}{3} \\
& A(y)=10 y+300
\end{aligned}
$$

Remplazando los valores anteriores en la ecuación 6 se obtiene el modelo matemático para el drenado del tanque el cual se rige por la ecuación 9.

$$
\frac{d y}{d t}=-34,77 \frac{y^{1 / 2}}{(10 y+300)}
$$

La ecuación 9 es una ecuación diferencial de primer orden no lineal de variables separables, la técnica para resolverla se estudió en la primera parte del curso de ecuaciones diferenciales y su solución general está dada por la ecuación 10.

$$
\frac{20}{3} y^{3 / 2}+600 y^{1 / 2}=-34,77 t+C
$$

Para obtener la solución particular de la ecuación 10 es necesario que los estudiantes planteen la condición inicial teniendo en cuenta el sistema real en estudio, en este caso el tanque inicia el proceso de drenado con una altura del agua de $12 \mathrm{~cm}$, por lo que $\mathrm{y}(0)=12$. Considerando esta condición inicial los estudiantes obtienen el valor de la constante $\mathrm{C}$ y de esta forma hallan un modelo matemático que relaciona las variables de entrada y salida del sistema en estudio, la altura del agua en el tanque $(y)$ y el tiempo (t) según se define en la ecuación 11.

$$
\frac{20}{3} y^{3 / 2}+600 y^{1 / 2}=-34,77 t+2355,59
$$

A partir de la ecuación 11 se obtiene el tiempo de drenado del tanque, cuando la altura del agua es y=0, simplemente reemplazando en la ecuación para obtener la ecuación 12.

$$
\frac{20}{3}(0)^{3 / 2}+600(0)^{1 / 2}=-34,77 t+2355,59
$$

De donde se obtiene finalmente el valor $\mathrm{t}=67.74 \mathrm{~s}$, que corresponde con el tiempo total para el drenado del tanque.

\section{Simulación}

Para obtener más información del modelo matemático los estudiantes realizaron la simulación del modelo en SIMULINK de MATLAB. El comportamiento gráfico de dicha simulación relaciona la variable de entrada y salida del modelo y el resultado se muestra en la Figura 5. Es posible obtener por medio del software MATLAB la información numérica del comportamiento del drenado del tanque en este caso se obtuvieron un total 50 puntos, algunos de ellos se muestran en la Tabla 2. 


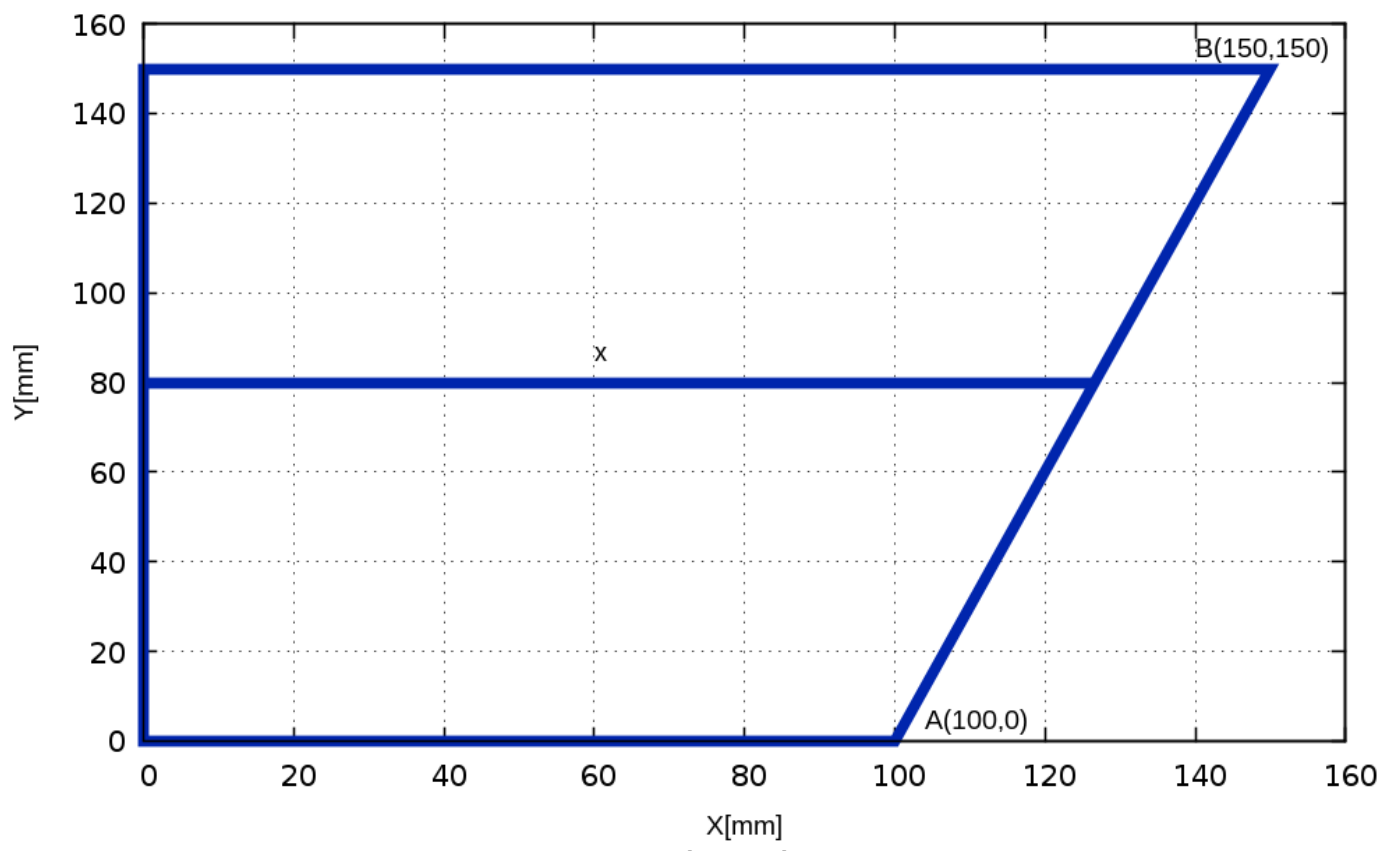

Fig. 4: Matematización del área transversal

Tabla 2: Datos obtenidos de la simulación.

\begin{tabular}{|l|l|l|l|l|l|l|l|l|l|l|l|}
\hline Altura [cm] & 12 & 11,20 & 10,03 & 7,79 & 5,40 & 4,18 & 3,34 & 2,83 & 2,12 & 0,26 & 0 \\
\hline Tiempo de drenado [s] & 0 & 2,81 & 7,00 & 15,40 & 25,20 & 30,80 & 35,00 & 37,80 & 42,10 & 58,80 & 67,74 \\
\hline
\end{tabular}

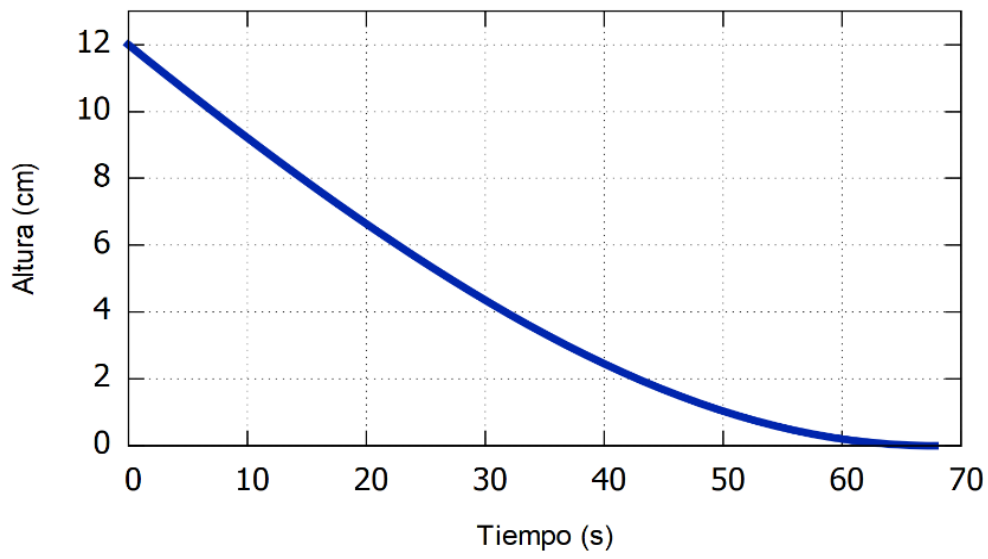

Fig. 5: Solución de la Ecuación Diferencial Utilizando Simulink de Matlab.

\section{Validación}

Para validar el modelo matemático los estudiantes obtuvieron datos experimentales del comportamiento del sistema utilizando el software TRACKER, en la Tabla 3 se muestran los datos experimentales y los datos obtenidos con el modelo matemático de las variables de entrada (altura del agua) y la variable de salida (tiempo de drenado) del sistema en estudio, al igual que la diferencia porcentual de los mismos, la cual es de $3,18 \%$ lo que indica que el modelo matemático propuesto es capaz de predecir dentro de unos límites razonables el comportamiento del sistema real. La gráfica de la información de la Tabla 3 se comparan con los resultados de la simulación en la Figura 6. 
Tabla 3: Diferencias porcentuales entre los datos del modelo matemático de caja blanca (MATLAB) y experimentales (TRACKER)

\begin{tabular}{|c|c|c|c|c|c|c|c|c|c|c|c|c|c|}
\hline $\mathrm{t}[\mathrm{s}]$ & 0 & 1,42 & 2,84 & 4,26 & 5,61 & 7,05 & 8,42 & 9,89 & 11,22 & 12,61 & 14,00 & 15,45 & 16,83 \\
\hline$Y_{\exp }[\mathrm{cm}]$ & 12 & 11,67 & 11,34 & 10,98 & 10,64 & 10,20 & 9,76 & 9,32 & 8,96 & 8,53 & 8,26 & 7,82 & 7,47 \\
\hline $\mathrm{Y}_{\mathrm{sim}}[\mathrm{cm}]$ & 12 & 11,60 & 11,20 & 10,81 & 10,42 & 10,03 & 9,65 & 9,27 & 8,89 & 8,52 & 8,16 & 7,79 & 7,44 \\
\hline dif \% & 0 & 0,58 & 1,21 & 1,62 & 2,07 & 1,66 & 1,11 & 0,52 & 0,82 & 0,08 & 1,17 & 0,41 & 0,05 \\
\hline $\mathrm{t}[\mathrm{s}]$ & 49,34 & 50,43 & 51,81 & 53,23 & 54,68 & 56,00 & 57,47 & 58,89 & 60,28 & 61,64 & 63,00 & 64,40 & 67,74 \\
\hline$Y_{\exp }[\mathrm{cm}]$ & 1,23 & 1,07 & 0,98 & 0,82 & 0,63 & 0,58 & 0,47 & 0,38 & 0,31 & 0,22 & 0,18 & 0,12 & 0,09 \\
\hline $\mathrm{Y}_{\mathrm{sim}}[\mathrm{cm}]$ & 1,15 & 0,99 & 0,84 & 0,70 & 0,57 & 0,46 & 0,36 & 0,27 & 0,19 & 0,13 & 0,08 & 0,04 & 0 \\
\hline $\operatorname{dif} \%$ & 1,15 & 1,01 & 3,57 & 5,71 & 5,71 & 3,51 & 4,35 & 5,56 & 7,41 & 5,26 & 7,69 & 0 & 0 \\
\hline
\end{tabular}

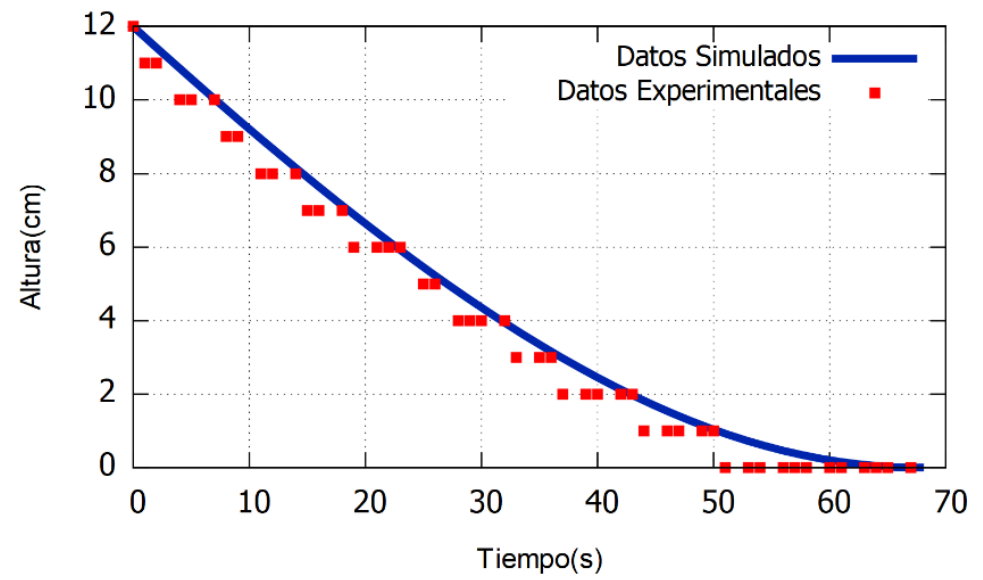

Fig. 6: Comparación grafica datos experimentales y simulados en drenado de tanque.

\section{Análisis de un modelo matemático de caja negra.}

En el desarrollo del modelo matemático de caja negra los estudiantes del curso de técnicas de variables de medición utilizaron un ajuste polinómico de segundo orden mediante el método de mínimos cuadrados. Los datos correspondientes al comportamiento del sistema se obtuvieron mediante el software TRACKER, de esta forma, el modelo matemático obtenido es: $y=0,002 t^{2}-0,3234 t+12,308$, cuyo coeficiente de ajuste $R^{2}$ es del 0 , 9988.Para obtener el tiempo de drenado del tanque se resuelve la ecuación de segundo orden cuando la atura del agua es $\mathrm{y}=0$, así el tiempo de drenado del tanque es igual a $61,15 \mathrm{~s}$.

\section{Comparación de resultados}

En la Tabla 4 se muestran los resultados del tiempo de drenado del modelo matemático de caja blanca, de caja negra y los del sistema (experimentales), así como la diferencia porcentual entre ellos.

Tabla 4: Resultados del tiempo de drenado obtenidos experimentalmente y por modelos matemáticos.

\begin{tabular}{|l|l|l|}
\hline & Tiempo de drenado [s] & \multirow{2}{*}{ Diferencia porcentual \% } \\
\hline Modelo matemático Caja Blanca & 67,74 & \\
\cline { 1 - 2 } Modelo matemático Caja Negra & 61,15 & \multirow{2}{*}{12,4} \\
\cline { 1 - 2 } Modelo matemático Caja Negra & 61,15 & \\
\hline Resultado experimental & 69,88 & \multirow{2}{*}{3,15} \\
\cline { 1 - 2 } Modelo matemático Caja Blanca & 67,74 & \\
\cline { 1 - 2 } Resultado experimental & 69,88 &
\end{tabular}




\section{ANÁLISIS Y DISCUSIÓN}

Este tipo de actividades permite que los estudiantes comprendan: el uso de las matemáticas como herramienta fundamental en el estudio de los fenómenos reales de ingeniería, las relaciones entre las variables, la formulación de modelos matemáticos, la importancia en el manejo del software para simular un fenómeno físico o para resolver un modelo matemático, entre otras. Los resultados del modelado matemático que realizaron los estudiantes permite evidenciar avances significativos en su proceso de formación y en el desarrollo de habilidades a la hora de utilizar las herramientas matemáticas, en éste caso cada grupo tiene una idea muy aproximada que les permite formular modelos de caja blanca y negra (según les corresponda), pues no sólo se estudiaron ejercicios de los libros, si no que en el proceso de modelado matemático fue necesario que analizarán el sistema, conocieran su comportamiento, determinaran distintas formas de abordarlo, y que se enfrentaran con las dificultades propias de un sistema real que dista de lo que ocurre en la teoría. En la etapa de validación, comprendieron la forma en que, como seres humanos nos aproximamos cada vez más al estudio detallado de los sistemas reales.

\section{CONCLUSIONES}

De acuerdo con el análisis y discusión de los resultados obtenidos en el presente estudio, se establecen las siguientes conclusiones:1) Debido a la necesidad actual de solucionar problemas reales de ingeniería se deben incluir técnicas de modelado matemático en los cursos de pregrado, de tal forma que los estudiantes se vayan habituando a analizar las problemáticas desde ésta perspectiva; 2) Es importante que los futuros ingenieros conozcan diferentes formas de modelar matemáticamente para que sean conscientes de los errores que se puedan cometer y en su quehacer profesional tengan el criterio suficiente para seleccionar la técnica más apropiada dependiendo de cada situación; 3) Éste tipo de ejercicios en el aula de clase permite a los futuros ingenieros interactuar con sistemas reales, problematizar situaciones y desarrollar actividades que los vinculan con su trabajo en el campo de ingeniería, les muestra distintas posibilidades para su desarrollo profesional y la importancia dominar las matemáticas para comprender y solucionar fenómenos físicos y de ingeniería; 4) Es imposible desarrollar tareas de modelado matemático sin tener una formación matemática sólida. 5) Los modelos matemáticos de caja negra son más imprecisos que los de caja blanca en la predicción de comportamientos de las variables de un sistema de ingeniería. 6) Este tipo de actividades permite que los estudiantes desarrollen habilidades de modelado matemático y así puedan relacionar los conceptos teóricos con los problemas de la vida real.

\section{REFERENCIAS}

ABET., Criteria for Accrediting Engineering Programs, ABET 415 N, Baltimore, USA (2017).

Alma, A.B., y García, M.L., Trabajos en Proyectos Como Herramienta Para Fortalecer las Habilidades Cognitivas en los Estudiantes, doi:10.4067/S0718-50062011000200004, Formación Universitaria, Vol. 4(2), 27-36 (2011).

Alpers, B., The Mathematical Modelling Competencies Required for Solving Engineering Statics Assignments. Mathematical Modelling and Applications: Crossing and Researching Boundaries in Mathematics Education, doi:10.1007/978-3-319-62968-1_16, Springer International Publishing, pp.189-199 (2017).

ASIBEI., Competencias y Perfil del Ingeniero Iberoamericano, Formación de Profesores y Desarrollo Tecnológico e Innovación, 1a Ed, ARFO Editores e Impresores Ltda, Valparaiso, Chile (2016).

ASEE., Transforming Undergraduate Education in Engineering Phase I: Synthesizing and Integrating Industry Perspectives, Report Arlington, USA (2013).

Casoli, P., Anthony, A. y Rigosi, M., Modeling of an Excavator System-Semi Empirical Hydraulic Pump Model. doi:10.4271/2011-01-2278, SAE International Journal of Commercial Vehicles. Sep 13;4(2011-01-2278):242-55 (2011).

Cellier, F.E., Continuous System Modeling, $1^{\text {a }}$ Ed.,3-17, Springer-Verlag, New York, USA (1991).

Chevallard, Y., La transposición didáctica. Del Saber Sabio Al Saber Enseñado. Buenos Aires: Aiqué (1991).

Cross, N., Engineering Design Methods: Strategies for Product Design. 3a Ed.,1-15, John Wiley \& Sons, The Atrium, England (1994).

Edwards, E. y Hamson, M, Guide to Mathematical Modelling, $1^{\text {a }}$ Ed,1-9, Macmillan Education Ltd, Londres, Inglaterra (1989).

Feisel, L y Rosa, A.J, The Role of the Laboratory in Undergraduate Engineering Education, doi:10.1002/j.2168-9830. 2005.tb00833.x, Journal of Engineering Education. 94 (1),121-130 (2005).

Frejd, P., Bergsten, C., Mathematical Modelling As A Professional Task, doi:10.1007/s10649-015-9654-7, Educational studies in mathematics. Jan 1;91(1):11-35 (2015).

Fritzson, P., Introduction to Modeling and Simulation of Technical and Physical Systems with Modelica, 2a Ed, 2-8, John Wiley \& Sons, USA (2011). 
Giraldo, A. y Pinilla, J. Simulación de Procesos de Negocios (BPSIM) Como Soporte Didáctico en el Aprendizaje de la Gestión de Procesos de Servicio, doi:10.4067/S0718-50062016000100011, Formación Universitaria Vol. 9(1), 99-108 (2016).

Leal, JJ., Cardona, JP., Agudelo, A., El Modelamiento Matemático Como Vía Idónea Para La Formación De Ingenieros, doi.org/10.14483/udistrital.jour.RC.2015.21.a9, Una reflexión pedagógica. Revista Científica. Jan 1;1(21):91-6 (2015).

$\mathrm{Li}$, T., Mathematical Modeling Education is the Most Important Educational Interface Between Mathematics and Industry; Educational Interfaces Between Mathematics and Industry. New ICMI Study Series, doi:10.1007/978-3-319-02270-3_5, Springer, Cham, vol 16, pp 51-58, New York, USA (2013).

Magana, A., Modeling and Simulation in Engineering Education: A Learning Progression. doi:10.1061/(ASCE)El.19435541.0000338. Journal of Professional Issues in Engineering Education and Practice. 143. 1-19 (2017).

McKenna, A., y Carberry, A. Characterizing the Role of Modeling in Innovation, International Journal of Engineering Education, ISSN:0949149X, 28(2), 263-269 (2012).

MEN., Resolución 2773 de 2003,1-3, Colombia, Bogotá (2003).

Mendible, A. y Ortiz, J., Modelización Matemática en la Formación de Ingenieros. La Importancia del Contexto, Enseñanza de la Matemática, ISSN:314-338,16 (1),133-150 (2007).

Minsky, M., Mind and Models. Massachusetts Institute of Tecnology,1-5 (1965).

Nirmala, K., Modeling Tools for Environmental Engineers and Scientists, ${ }^{\text {a } E d, 1-9, ~ C R C ~ P r e s s ~ L L C, ~ W a s h i n g t o n, ~ D . C, ~}$ USA (2002).

Nugroho, L., y Akmeliawati, R. Comparison Of Black-Grey-White Box Approach In System Identification Of A Flight Vehicle, doi :10.1088/1742-6596/1130/1/012024, JPhCS 1130, no. 1 (2018).

Preziosi, L., y Bellomo, L.N, Modelling, Mathematical Methods and Scientific Computation, $1^{\mathrm{a}}$ Ed, CRC Press, Florida, USA (1994).

Shier, D.R., y Wallenius, K.T, Applied Mathematical Modeling a Multidisciplinary Approach, $1^{\text {a }}$ Ed, 5-9, Chapman \& Call/CRC, New York, USA (1999).

Shiflet, A.B y Shiflet, W.G, Modeling and Simulation for the Sciences, 2a Ed.,1-20, Princeton University Press, New Jersey, USA (2014).

Stadnicka, D., Litwin, P., y Antonelli, D., Human Factor In Intelligent Manufacturing Systems: Knowledge Acquisition And Motivation, doi:10.5937/fmet1904823S, FME Transactions. 47(1),823-830 (2019).

Van Can, H.J., Te Braake, H.A., y otros 3. Understanding And Applying The Extrapolation Properties Of Serial Gray-Box Models, https://doi.org/10.1002/aic.690440507, AIChE journal. May;44(5):1071-89 (1998).

Velten, K., Mathematical Modeling and Simulation: Introduction for Scientists and Engineers, $1^{\mathrm{a}}$ Ed, 1-46, Verlag GmbH \& Co. KGaA, Strauss GmbH, Morlenbach, Alemania (2009).

Venkateswarlu, P., Establishing a Centre for Engineering Experimentation and Design Simulation: A Step Towards Restructuring Engineering Education in India, doi:10.1080/03043797.2016.1158794, European Journal of Engineering Education, Vol. 42(4),349-367 (2017).

Wang, X.G., Zou, Z.J., y otros 2. W., System Identification Modeling Of Ship Manoeuvring Motion in 4 Degrees Of Freedom Based on Support Vector Machines, doi:10.1007/s13344-015-0036-9, China Ocean Engineering. Jun 1;29(4):519-34 (2015).

Yilmaz, L., Reproducibility in M\&S Research: Issues, Strategies and Implications for Model Development Environments, doi:10.1080/0952813x.2012.693842, Journal of Experimental and Theoretical Artificial Intelligence, 24(4), 457474 (2012).

Zawojewski, J.S., Dux, D.H. y Bowman, K., Models and Modeling in Engineering Education Designing Experiences for All Students, $1^{\text {a }}$ Ed., 1-6, Sense Publishers, Rotterdam, The Netherlands (2008).

Zeigler, B.P., Muzy A. y Kofman, E., Theory of Modeling and Simulation, 3ªEd.,29-35, Academic Press, San Diego, USA (2019).

Zendehboudi, S., Rezaei, N., Lohi, A., Applications of Hybrid Models in Chemical, Petroleum, and Energy Systems: A Systematic Review, https://doi.org/10.1016/j.apenergy.2018.06.051, Applied energy. Oct 15;228:2539-66 (2018). 